Special issue in honor of Prof. George C. Papageorgiou

\title{
An open Internet of Things (IoT)-based framework for feedback control of photosynthetic activities
}

\author{
S. YUAN ${ }^{*}$, H. TANG ${ }^{* *}$, L.J. FU*, J.L. TAN**, G. GOVINDJEE*, and Y. GUO ${ }^{*, * *,+}$ \\ Key Laboratory of Advanced Process Control for Light Industry, Ministry of Education, School of IoT, \\ Jiangnan University, 214122 Wuxi, China* \\ Lushixin Sci. \& Tec. (Wuxi) Co. Ltd., 214124 Wuxi, China ${ }^{* *}$ \\ Department of Bioengineering, University of Missouri, Columbia, MO 65211, USA $A^{* * *}$ \\ Center of Biophysics \& Quantitative Biology, Department of Biochemistry and Department of Plant Biology, \\ University of Illinois at Urbana-Champaign, Urbana, IL 61801, USA
}

\begin{abstract}
Active control of photosynthetic activities is important in plant physiological study. Although models of plant photosynthesis have been built at different scales, they have not been fully examined for their application in plant growth control. However, we do not have an infrastructure to support such experiments since current plant growth chambers usually use fixed control protocols. In our current paper, an open IoT-based framework is proposed. This framework allows a plant scientist or agricultural engineer, through an application programming interface (API), in a desirable programming language, (1) to gather environmental data and plant physiological responses; (2) to program and execute control algorithms based on their models, and then (3) to implement real-time commands to control environmental factors. A plant growth chamber was developed to demonstrate the concept of the proposed open framework.
\end{abstract}

Keywords: chlorophyll fluorescence; greenhouse; Internet of things; open framework; photosynthesis model; plant growth chamber.

\section{Introduction}

Increasing human population, decreasing land area for cultivation, and changes in weather conditions have led to a

\section{Highlights}

- An open IoT-based framework for feedback control of photosynthetic activities was proposed

- Extended Kalman filter was employed to demonstrate the estimation of plant physiological state

- A plant growth chamber was developed to demonstrate the proposed framework global food shortage on our Earth (Long et al. 2015, Chang and Zhu 2017, Christensen et al. 2018, Dinar et al. 2019). Active control of plant growth activities is important for both plant growth study and biomass production, which is,

Received 16 August 2021

Accepted 7 December 2021

Published online 20 January 2022

${ }^{+}$Corresponding author

e-mail: guoy@missouri.edu, guoy@jiangnan.edu.cn

Abbreviations: API - application programming interface; CRC - cyclic redundancy check; EKF - extended Kalman filtering; ETR electron transport rate; IoT - Internet of things; MIAPPE - minimum information about a plant phenotyping experiment; MPC - model predictive control; MQTT - Message Queuing Telemetry Transport; PID - proportional, integral, and derivative; $\Phi_{\text {PSII }}-$ effective quantum yield of PSII photochemistry.

Acknowledgments: This project was partially supported by the National Natural Science Foundation of China (No. 51961125102, 31771680, 31871840), and the 111 Project (B12018). For IT support, Govindjee personally thanks the staff of the Life Sciences Office of Information Technology at the University of Illinois at Urbana-Champaign.

Conflict of interest: The authors declare that they have no conflict of interest. 
worldwide, one of the 125 important and difficult problems (Kennedy and Norman 2005). Practical photosynthesis dynamic models are important for plant growth strategy development, which have been developed, in the past, at different scales. For example, Zhu et al. (2005) used first-order reaction kinetics to model photosynthetic activities, while Guo and Tan (2011) used second-order reaction kinetics. Related studies involving mathematical modeling and simulation of photosynthesis have been summarized by Stirbet et al. (2014). Marshall-Colon et al. (2017) used multi-scale models to facilitate the simulation of the entire plant. Feng et al. (2018) developed a sevenstate chlorophyll fluorescence model for photosystem II activities, and Kannan et al. (2019) evaluated the modeling process from gene expression to photosynthetic metabolism. Further, Fu et al. (2020) evaluated the state of photosynthesis by a model, which included the extended Kalman filtering method, and used the estimated chlorophyll fluorescence as feedback to establish a light intensity feedback regulation model. However, these models have not yet been tested for their applicability in plant growth control experiments, which might explain why the current greenhouse control strategy is still not based on information from the physiology of plants and does not reflect real-time plant photosynthetic needs. At present time, there is a lack of available infrastructure to support such 'controlled experiments' since current plant growth chambers and greenhouse platforms usually use fixed protocols and do not allow one to compile plant growth strategies based on plant physiological information and photosynthesis models. Further, it is quite time-consuming to rebuild the whole system to test updated photosynthetic activity control strategy. These facts unavoidably hinder important interdisciplinary research. We discuss below the means to overcome these problems.

To facilitate photosynthetic activity control research, there is an urgent need to develop an open platform that would allow plant scientists and agriculture engineers to build their photosynthetic activity control strategy by using their 'desired' programming language. Internet of Things (IoT) is a solution to achieve this goal; it senses, transmits, stores, and processes information and controls targets (Balaji et al. 2018, Verdouw et al. 2019, Astill et al. 2020, Malik et al. 2020). An IoT-based platform plays the role of data display and user control in the system for greenhouses as well as for plant growth chambers; further, it plays an essential role in the field of 'smart agriculture'. Although there has been some research on the open IoT framework that allows reprogramming (Hemming et al. 2019), there are only a few open IoT framework applications in the field of agriculture, especially for photosynthetic activity control. At this time, some industry-driven robust solutions, such as LamnaTec (Kim 2020) and Photon Systems Instruments platforms (Yu et al. 2015) are not open for users to obtain real-time data, and to execute control strategies through API. Further, researchers need to use specific software to write control strategies, which is not conducive to real-time control of photosynthesis. Although there are some community supported solutions, such as Think Speak (Pasha 2016) and
Amazon Web Services (AWS) (Bermudez et al. 2013), yet for researchers who do not know much about the sensors and the actuators, it is still a challenge to build a complete growth chamber system using such a communitysupported solution.

In this work, we propose an open framework to realize the photosynthetic activity control experiments, which allows customizing control strategy according to a photosynthesis model. Then we demonstrate how to use it conveniently by developing and constructing a plant growth chamber based on the proposed open IoT-based framework. The proposed open framework and the developed plant growth chamber can not only control real-time environment parameters and control directly measurable plant physiological variables but also control indirectly measurable variables through a photosynthesis dynamic model.

\section{Development of an open IoT-based framework for feedback control of photosynthetic activities}

Overall design and integration of the IoT framework: To build the open framework, based on the traditional three-layer IoT framework (Mahmoud et al. 2015), there is a need to add an open resource layer to make photosynthetic activity control experiments, as well as secondary development possible. As shown in Fig. 1, the proposed framework has four layers: (1) a device layer; (2) a network layer; (3) an open resource layer; and (4) an application layer.

\section{(1) Device layer}

The device layer is located at the very bottom of the framework. This layer includes a sensor (Fig. 1, left) and actuator (Fig. 1, right) devices for environment parameters, and for the sensing of plant physiological status and for controlling environment parameters, as well as for the control boards. In the device layer, information is transmitted between the sensors, the actuators, and the control board through industry standard protocols RS485. Users can also view the ModBus code at: https:// github.com/Jiangnan-IOT-C426/OpenCode/tree/master/ STM32PlantChamber/Drivers/Modbus.

\section{(2) Network layer}

The network layer is responsible for wireless data transmission. These network devices are divided into client devices (connected to the control board or clientside network devices) and server devices (deployed on the cloud server). If client-side users need to gather and control environment parameters on a remote device, they need to ensure that the remote device can access the Internet, and can establish a connection with the greenhouse or a growth chamber.

\section{(3) Open resource layer}

The open resource layer is the core of shared device resources and provides the user cross-platform inter- 


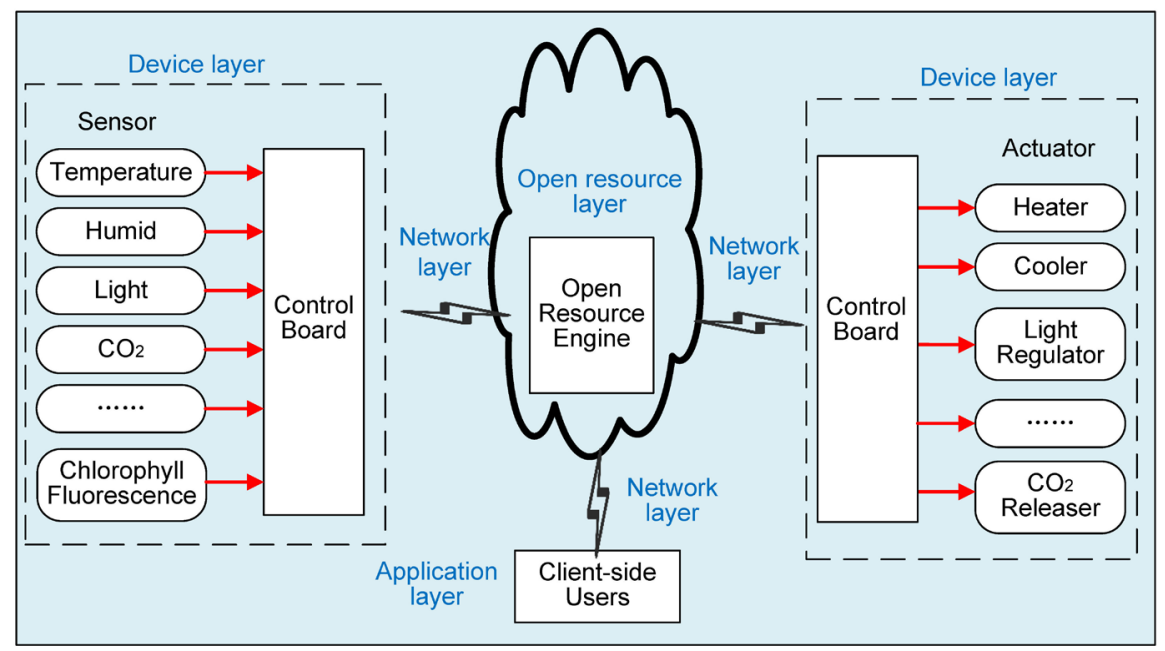

Fig. 1. The proposed open IoT-based framework for feedback control of photosynthetic activities.

connection through the API files, as explained in section 'Designs of API files in the open framework'. This layer provides some basic functions, such as physical device management, device data storage, service authority management, and open resource control. The most important role for the resource layer is to ensure that client-side users can use different programming languages to establish a connection with a greenhouse or a growth chamber.

\section{(4) Application layer}

The application layer is directly useful for both the plant scientists and the framework managers. It contains various applications, such as websites and mobile apps; it is mainly responsible for data visualization and distribution of the API files. Plant scientists can use this layer to view environment parameters during the entire cycle of plant growth; they must also obtain the API files corresponding to the programming language from the framework managers, and use this layer to complete the download of all the API files.

Designs of API files in the open framework: The open resource layer structure is shown in Fig. 2. First, the physical device management system (see Supplement $1 A$ ) abstracts and registers the physical devices in a plant growth chamber or a greenhouse. Second, through the device data storage (see Supplement $1 B$ ), the requested sensor data and the control commands, with successful cyclic redundancy check (CRC) checks, are then sent to the user and actuators. Third, the users use the functions in the API file in open resource control (see Supplement $1 C)$ to gather the sensor data and the control environment parameters. Fourth, and the last, the service authority management system (see Supplement 1D) checks the authority of the API and intercepts all 'illegal' uses.

Users may realize customized photosynthetic activity control strategy through the API file as follows. First, they can use the provided API file that corresponds to their desired programming language and put the API



Fig. 2. The open resource layer structure.

file under the control algorithm path. Second, they can visit sensor data (environmental variables, plant growth status, and physiological variables, depending upon the sensors included in the system) by using the provided functions in the API file. An example of data query commands and descriptions is available in Supplement 2. Third, they can write the photosynthetic activity control algorithm based on their photosynthesis models by using the desired computer language, and finally 'call' the actuators through corresponding functions in the API file based on real-time computation. An example of control commands and descriptions is available in Supplement 3. The photosynthetic activity control process through the API file is shown in Fig. 3.

Three typical customized control strategies at the user side: As stated above, users have full flexibility to write a plant control algorithm based on a particular model. Here, three common scenarios of control strategies based on this open IoT framework are provided: (1) real-time environment parameter control without a plant physiological response as feedback; (2) target plant 
physiological response, which is directly measurable; and (3) target plant physiological response, which is not directly measurable.

\section{Control scenario 1: Only control environment parameters}

In photosynthetic activity control, sometimes it is necessary to maintain or change some environmental parameters, such as temperature, light intensity, and carbon dioxide concentration (Kramer 1981, Bulthuis 1987, Barber and Andersson 1992). Fig. $4 A$ shows the flow chart of realtime control of these environment parameters; here, the input represents the environmental parameters that need to be controlled and can represent a series of natural environmental conditions. On the other hand, the output includes all the expected environmental parameter outputs, such as temperature, light intensity, and carbon dioxide. Further, the controller refers to the customized control strategy, the actuator being the machine to realize all the

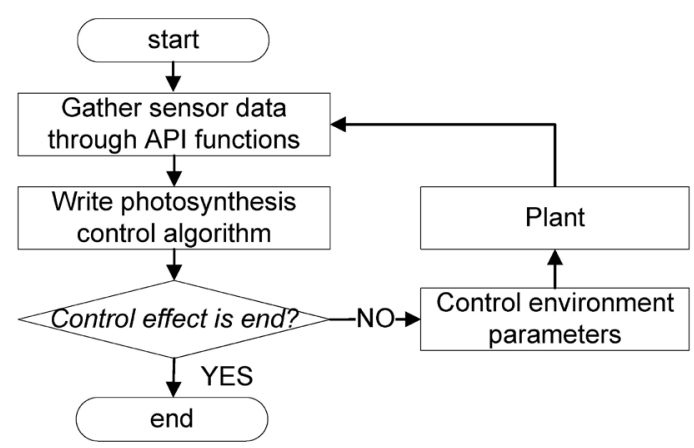

Fig. 3. The photosynthetic activity control process through the API file. control commands, and the plant is the object that needs to be controlled.

Through this open framework, described above, this type of plant growth control experiment can be implemented by the following simple codes.

\section{Control_Operator(Parameter_target)}

The Control Operator means control command, the Parameter includes all the environmental parameters that need to be controlled, and the target is used for the values of all the environmental parameters that need to be controlled.

\section{Control scenario 2: Target plant physiological response is directly measurable}

Ideally, plant photosynthetic activity control is based on the real-time physiological response of plants. For the case of the response, which is measurable, a flow chart of the control scheme is shown in Fig. $4 B$. Here, the measure is the plant physiological response from the sensors. The difference (in Fig. 4B) is the bias between the input target and the measured plant physiological response.

This type of plant control experiment can be implemented by the following code (see below): $n$ is the $n$-th control moment, Select Sensor is for the select command, and, Parameter is the plant physiological response. Based on the difference at each control moment, a difference-based control strategy can be applied.

$$
\begin{aligned}
& \text { measure }(n)=\text { Select_Sensor }(\text { Parameter }) \\
& \text { difference }(n)=\operatorname{input}(n)-\text { measure }(n) \\
& \text { Control_Operator(Parameter_target })=\mathrm{F}_{1}[\text { difference } \\
& (i, \ldots, n-1, n)]
\end{aligned}
$$

where $i$ is the $i$-th control moment in the past, difference $(i, \ldots$, $n-1, n)$ is the difference from $i$-th to $n$-th control moment,

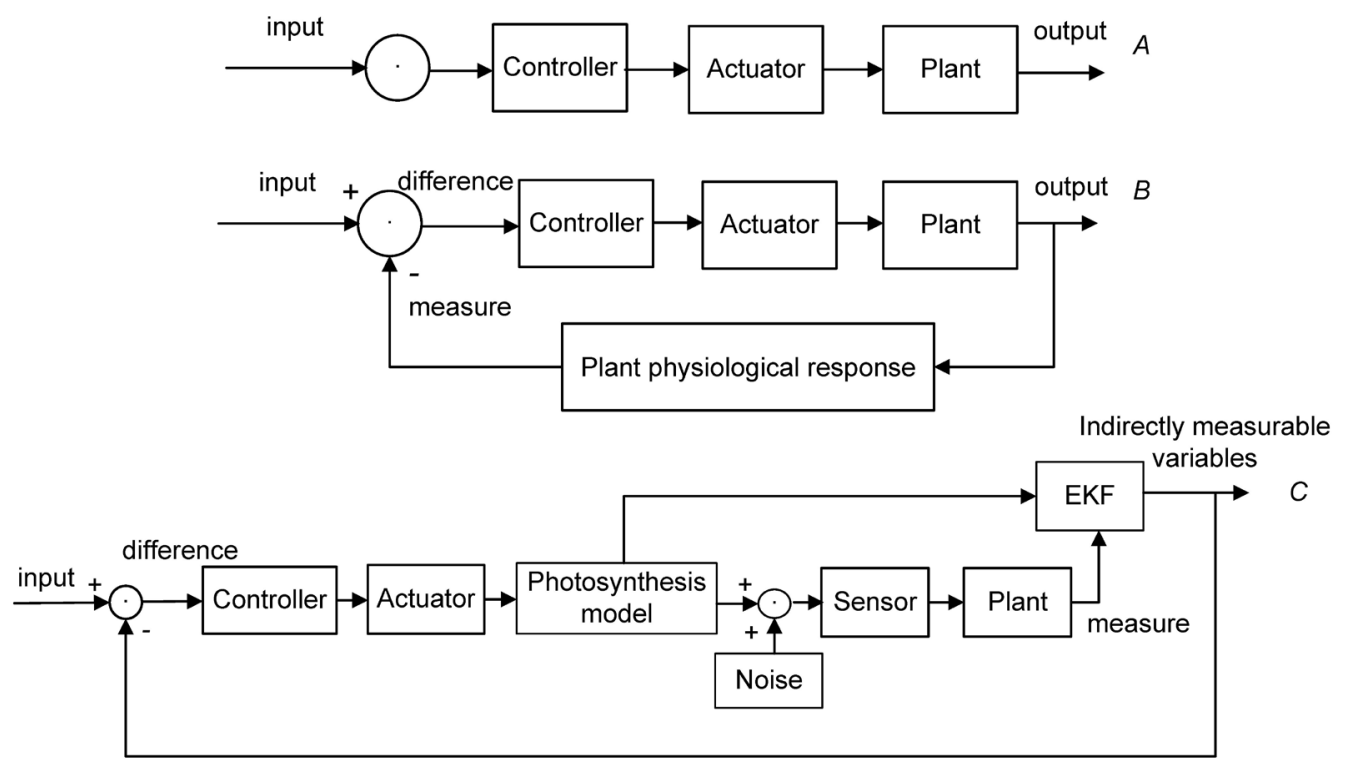

Fig. 4. Flow chart of three typical customized control strategies. 
and $F_{1}$ represents the control function that responds to the difference $(i, \ldots, n-1, n)$.

\section{Control scenario 3: Target plant physiological response is not directly measurable}

Sometimes, target plant physiological responses cannot be measured due to the lack of real-time sensors. However, these responses may couple with some measurable outputs through a mathematical model (Guarini and Moritz 2009). The control of these unmeasurable target plant physiological responses may be realized by estimating the unmeasurable response and updating the model parameters through real-time data, usually using the technique of extended Kalman filtering (EKF) (Campani et al. 2019). A flow chart of this type of control is shown in Fig. $4 C$.

This type of plant growth control experiment can be implemented by the following codes:

$$
\begin{aligned}
& x_{\text {pred }}(n)=F(n) \hat{x}(n-1)+B u(n-1) \\
& p_{\text {pred }}(n)=F(n) P(n-1) F^{T}(n)+Q(n-1) \\
& K(n)=\frac{p_{\text {pred }}(n) H^{T}(n)}{H(n) p_{\text {pred }}(n) H^{T}(n)+R(n)}
\end{aligned}
$$

where $n$ represents the $n$-th control moment; $n-1$ is for the $(n-1)$-th control moment; $x_{\text {pred }}(n)$ is the predicted value of $\hat{x}(n-1) ; F(n)$ is the Jacobian matrix of the partial derivative of the photosynthesis model to the state vector; $\hat{x}(n-1)$ is the best estimate of indirectly measurable variables for the $(n-1)$-th control moment; $B$ is the gain matrix of input; $u(n-1)$ is the output of the actuator. Further, $p_{\text {pred }}(n)$ is the prediction covariance matrix; $P(n-1)$ is the covariance matrix of $\hat{x}(n-1) ; Q(n-1)$ is the random noise covariance matrix; $K(n)$ is the Kalman filter gain matrix; $H(n)$ is the Jacobian matrix of the partial derivative of the observation model to the state vector; and $R(n)$ is the measurement noise covariance matrix. In addition to the above, we have:

$$
\begin{aligned}
& \hat{x}(n)=x_{\text {pred }}(n)+K(n)\left[Z(n)-H(n) x_{\text {pred }}(n)\right] \\
& P(n)=[\mathrm{I}-K(n) H(n)] p_{\text {pred }}(n)
\end{aligned}
$$

where $\hat{x}(n)$ is the best estimate of indirectly measurable variables for $n$-th control moment; $Z(n)$ is the real-time data from the sensors; $P(n)$ is the covariance matrix of $\hat{x}(n)$; and $I$ is the identity matrix. Based on the difference at each control moment, a difference-based control strategy can be used to control $\hat{x}(n)$, as shown below.

$\operatorname{difference}(n)=\operatorname{input}(n)-\hat{x}(n)$

Control_Operator(Parameter_target $)=\mathrm{F}_{2}[$ difference $(i, \ldots$, $n-1, n)]$

where $F_{2}$ is control function that responds to the difference $(i, \ldots, n-1, n)$. We note that the above codes can be changed according to different difference calculation methods.

\section{Experiments}

A plant growth chamber design based on the proposed open framework: To illustrate the concept of the proposed open framework, we have developed and constructed a plant growth chamber. This chamber is an accurately controlled small greenhouse and can be used for feedback control experiments of plant growth, based on environmental and plant physiological responses.

The plant growth chamber and core devices are shown in Fig. 5. Environmental sensors are deployed on top of the growth chamber (see Fig. $5 A$ ). A temperature sensor, a humidity sensor, and a light sensor are integrated into one transmitter (1); (2) is an interface for image collection; and (3) is a carbon dioxide sensor. In addition to environmental sensors, a chlorophyll fluorescence sensor (4) connected to the board to measure the photosynthetic activities of the plants being studied. A fan is placed in the growth chamber to ensure that the concentration of the gas in the chamber quickly reaches a uniform value, and the readings of the gas sensors are correctly recorded.

The external structure of the plant growth chamber is shown in Fig. $5 B$. In the plant growth chamber, there are actuators to control temperature, light intensity, and carbon dioxide concentration. In our system, light intensity is controlled by LEDs, which provide a wide range of light intensities (1). The carbon dioxide $\left(\mathrm{CO}_{2}\right)$ concentration control system includes a $\mathrm{CO}_{2}$ gas release actuator connected to a $\mathrm{CO}_{2}$ tank (2)). The temperature control system includes both the heater and the cooler actuators in the form of electromagnetic relays (3) and (4). Detailed plant growth experiment process and wireless transmission method are shown in Supplement 4. A detailed description of each sensor and actuator can be found in Supplement 5.

Implementation examples of the three typical customized photosynthetic activity control strategies: Here, we have provided three typical customized photosynthetic activity control strategies, which correspond to the three control scenarios, shown in Fig 4. Further, we have given here three control examples to call the functions in the API files by using Java, Python, and Matlab in the supplementary file and the codes have been uploaded at:
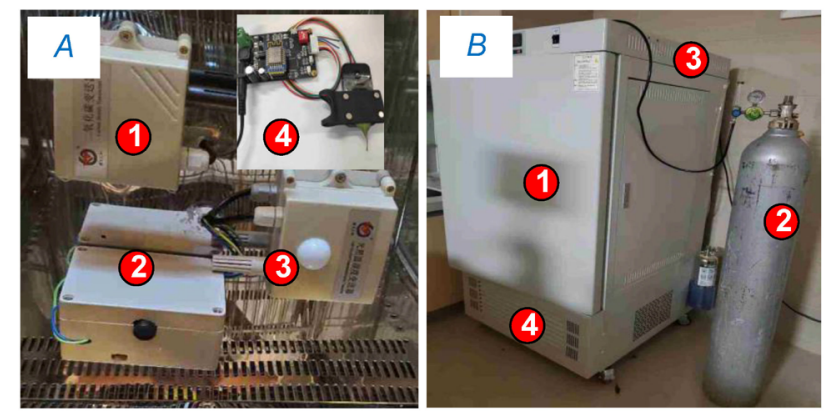

Fig. 5. The plant growth chamber and core devices. In Fig. 5A, 1 - transmitter of a temperature sensor, humidity sensor, and light sensor; 2 - interface for image collection; $3-\mathrm{CO}_{2}$ sensor; $4-$ chlorophyll fluorescence sensor. In Fig. 5B, 1 - LEDs; $2-\mathrm{CO}_{2}$ gas release actuator; 3 - heater actuator, 4 - cooler actuator. 
https://github.com/Jiangnan-IOT-C426/OpenCode/tree/ master/CaseStudies.

\section{Control example 1: Control environment temperature and $\mathrm{CO}_{2}$ concentration}

In conducting control experiments, the most important thing was to control both the temperature and the concentration of $\mathrm{CO}_{2}$. Here, we used the Java language for the temperature control and the Python language for the $\mathrm{CO}_{2}$ control as examples to demonstrate that different programming languages can be used on the platform. The temperature control strategy, used here, adopts threshold control. If the current temperature is lower than the set temperature, the heater is turned on; otherwise, the refrigerator is turned on. The control result is shown in Fig. $6 \mathrm{~A}$.

Starting from the initial value, $\mathrm{CO}_{2}$ concentration is increased every $100 \mathrm{~s}$ to achieve the different concentrations of $\mathrm{CO}_{2}$, used in this study. The control result is shown in Fig. $6 \mathrm{~B}$. For this growth chamber, the function of lowering $\mathrm{CO}_{2}$ has not been added, but this can be easily done by blowing air or $\mathrm{N}_{2}$ into the chamber.

\section{Control example 2: A closed-loop chlorophyll $a$ fluorescence control experiment}

Chlorophyll $a$ fluorescence is a sensitive measure of the photosynthetic process (Govindjee et al. 1986, Baker 2008, Fernandez-Jaramillo et al. 2012, Walker et al. 2018). Here we use it as an example to show how to control a
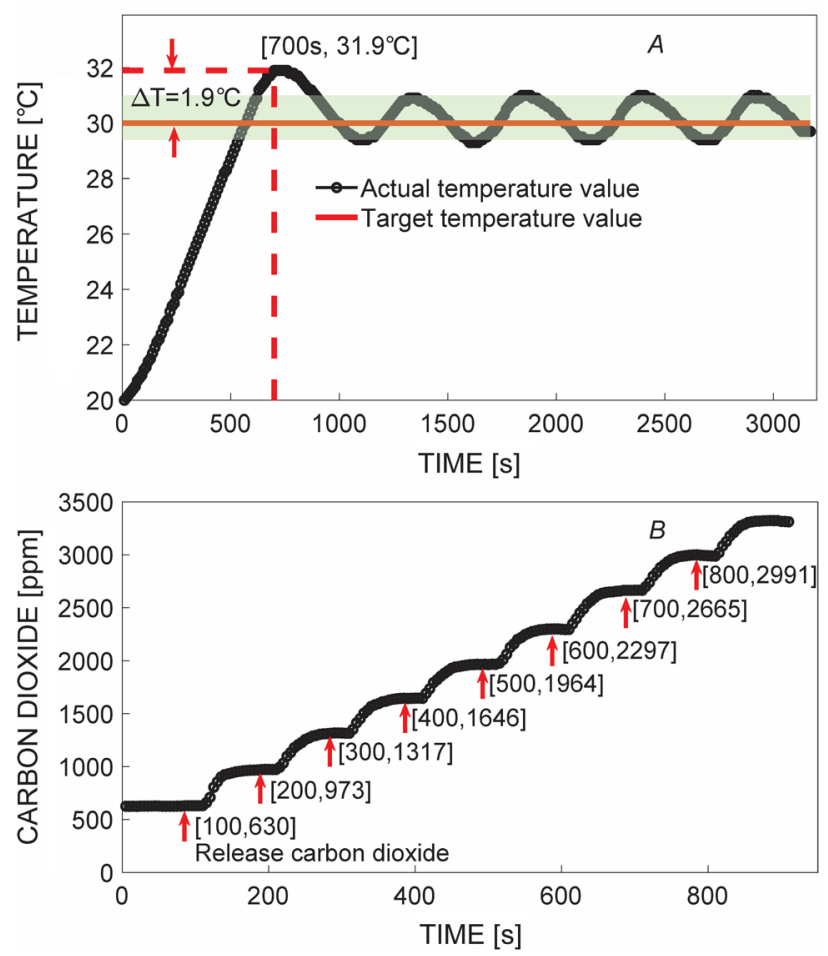

Fig. 6. Real-time environmental parameters control results. measurable target in the photosynthetic process. We note that chlorophyll $a$ fluorescence only serves as an example; the following method is equally applicable for other measurable targets in similar cases. We use here Matlab language for our continuous chlorophyll $a$ fluorescence tracking experiment. The API was already used to obtain measured chlorophyll $a$ fluorescence induction curves as well as to control the excitation light intensity to achieve our target chlorophyll $a$ fluorescence signal.

Here, we used the proportional, integral, and derivative (PID) closed-loop control algorithm in the experiment presented (controller). Chlorophyll fluorescence data (plant physiological response), measured in real time, was used as feedback to control the excitation light intensity (actuator), which changes its fluorescence induction (see e.g., Padhi et al. 2021). The control strategy is shown below.

$$
\operatorname{difference}(n)=\operatorname{input}(n)-\text { measure }(n)
$$

where $n$ represents the $n$-th time, difference $(n)$ is the measurement difference at $n$-th moment, $\operatorname{input}(n)$ is the target value, and measure( $n)$ is the chlorophyll $a$ fluorescence from the sensor.

$$
\begin{aligned}
& u(n)=k_{p} \text { difference }(n)+k_{i} e(n) \\
& e(n)=\operatorname{difference}(1)+\operatorname{difference}(2)+\ldots+\operatorname{difference}(n-1)
\end{aligned}
$$

where $k_{p}$ and $k_{i}$ are PID control parameters; only PI control is used here; $u(n)$ is the fluorescence excitation light intensity controlled by the actuator and refers to light that is used to produce chlorophyll fluorescence, and $e(n)$ is the cumulative difference of the positional PID control. Fig. 7. shows the experimental result for controlling chlorophyll $a$ fluorescence intensity.

\section{Control example 3: A model-based closed-loop $Q_{A}{ }^{-}$ control experiment}

In photosystem II, the primary electron acceptor plastoquinone $\mathrm{Q}_{\mathrm{A}}$ is reduced to $\mathrm{Q}_{\mathrm{A}}^{-}$once it receives an electron from the reaction center of PSII. The intensity of chlorophyll $a$ fluorescence is closely related to the redox state of $\mathrm{Q}_{\mathrm{A}}$; for further background on $\mathrm{Q}_{\mathrm{A}}$ and its relation to chlorophyll a fluorescence, see Dietz et al. (1985), Shinkarev and Govindjee (1993), and Schansker et al. (2014). Although it is well established that complete reduction of $\mathrm{Q}_{\mathrm{A}}$ leads to maximum chlorophyll $a$ fluorescence and the ratio between the variable chlorophyll $a$ fluorescence and the maximum chlorophyll $a$ fluorescence accounts for the quantum efficiency of PSII photochemistry, Sipka et al. (2021) have pointed out that this is not the case but the fluorescence ratio should rather be considered an empirical parameter. However, this controversy does not affect using chlorophyll $a$ fluorescence to demonstrate the concept of the framework. $\mathrm{Q}_{\mathrm{A}}^{-}$cannot be directly measured by the existing sensors, but it can be estimated indirectly through chlorophyll $a$ fluorescence in a plant photosynthesis model (see e.g., Zhu et al. 2005).

Here, we have used a model (a photosynthesis model) based on seven state variables, as presented by Feng et al. 

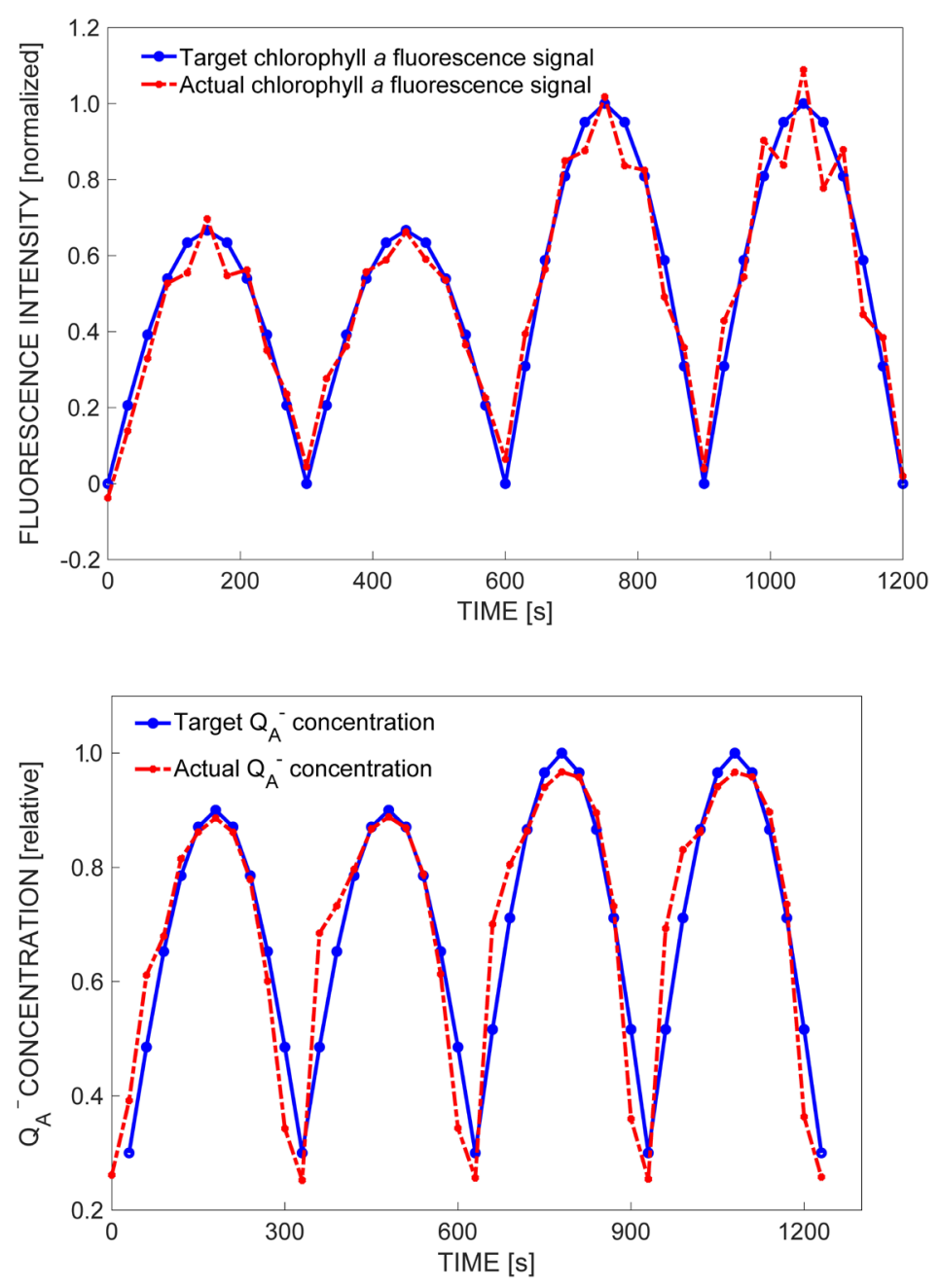

Fig. 7. A closed-loop chlorophyll $a$ fluorescence control experiment results.
(2018), to demonstrate how to control $\mathrm{Q}_{\mathrm{A}}^{-}$(indirectly measurable variable) in real time. Chlorophyll fluorescence data (sensor) was used for EKF to control the excitation light intensity (actuator). By substituting the model into the third scenario, a PID control strategy (controller) was applied to get the control result, shown in Fig. 8.

\section{Discussion}

In this work, we have proposed an open IoT-based framework for feedback control of photosynthetic activities. The functions for calling sensors and actuators are packaged in the API files. Users may use any computer language that supports internet communication to call these functions. Most current computer languages support internet communication functions. We have provided three control examples to call the functions in the API files by using Java, Python, and Matlab. Sample codes for the platform are hosted (and available) at https://github. com/Jiangnan-IOT-C426/OpenCode as a reference; thus, anyone can build such a chamber using this information. The work, presented in this paper, clearly shows the flexibility of the proposed open framework for plant growth experiments, especially for customized control strategy based on a photosynthesis dynamic model. Models can be easily integrated into a control strategy and executed by the framework. Iersel et al. (2016a,b) have proposed a feedback control method based on chlorophyll $a$ fluorescence to maintain a stable electron transfer rate (ETR) and photochemical quantum efficiency $\left(\Phi_{\text {PSII }}\right)$ by changing the excitation light. The values for ETR and $\Phi_{\text {PSII }}$ were derived from both the maximum (peak) and steady-state chlorophyll $a$ fluorescence values (see e.g., Genty et al. 1989). This effort was very important in controlling plant growth activities; however, this earlier system was not open to allow the users to write and implement users' customized control algorithms to test a photosynthesis model in a growth chamber or for controlling plant growth in real production by using different protocols. The proposed framework, in our current work, allows one to replace photosynthesis models at the user end, and thus provides a versatile platform to test the performance of a plant model and growth strategy experimentally.

The plant growth chamber and experiments in this work illustrate the usability of the open framework. If the devices do not have standard communication ports, open framework developers may encapsulate them as standard 
communication ports to achieve information acquisition and actuator control. However, users may need to calibrate the sensors, when needed, using the instructions from the sensor producers. Morphological measurement techniques such as 3D scanning and fluorescence imaging can also be used to evaluate the growth of entire plants. If and when the users would use morphological measurement technology in the overall framework for plant growth control, a morphologic pattern recognition algorithm could be deployed in the cloud server or on a local computer to automatically extract plant growth status information and, then to feed this information to a plant growth model that includes the growth status information as a model term or as a variable to develop a corresponding control strategy based on the chosen model as well as information from the imaging system.

For wireless transmission protocols, in our work, we have used the Socket protocol (Juhasova et al. 2017). Instrument developers can encapsulate the Socket protocol with application-layer programs, such as Message Queuing Telemetry Transport (MQTT) protocol (Light 2017) and REST API (Zhou et al. 2014). In future work, the data storage unit can be rebuilt to make it compatible with established minimum information standards, such as minimum information about a plant phenotyping experiment (MIAPPE) (Pommier et al. 2018). Some typical control strategies can be encapsulated in future work to reduce users' efforts in the development of plant growth algorithms. If a user model is used for plant production improvement, it is also desirable to adopt specific control strategies, such as in Model Predictive Control (MPC). We note that many dynamic factors make photosynthetic regulation a highly challenging process. A meaningful way to understand this process is to examine how photosynthetic activities are regulated under a simulated natural environment, which changes much more slowly as compared to the time lag of the chamber response. Therefore, it may not be a problem to develop a control strategy to mimic photosynthetic control under natural environment, but by using corresponding plant physiological models, control algorithms, sensors, and actuators. In this way, plant growth under the natural environment can be observed, which would be very meaningful considering the uncertainties brought by global warming. This would be very important for dealing with future agriculture production.

The focus of our current paper was to propose a platform for easy implementation of a control strategy, based on different photosynthetic models, instead of providing an optimal photosynthesis model. Although the biochemical test experiments were not conducted in this work, it does not affect our demonstration of the function and important use of the IoT platform. This platform is expected to serve as a standard for future plant growth chambers.

Conclusions: In this work, the design and implementation of an open IoT-based framework for photosynthetic activity control are presented and discussed. A plant growth chamber was developed based on the proposed open framework concept to demonstrate its usefulness for three scenarios: (1) strategy for environmental parameter control without a model, (2) a non-model-based control strategy for plant physiological variable that is measurable, and (3) a model-based control strategy for plant physiological variable that is not measurable. Based on the work presented here, plant scientists, as well as agricultural engineers, can write control algorithms in their preferred language by employing different photosynthetic model structures and by connecting different sensors and actuators. The open framework, presented in this work, provides flexibility on plant growth protocols to plant scientists and agricultural engineers without having them rebuild the entire hardware and software. This framework is expected to facilitate photosynthesis control research and the expansion capabilities of agriculture IoT facilities.

\section{References}

Astill J., Dara R.A., Fraser E.D.G. et al.: Smart poultry management: Smart sensors, big data, and the internet of things. - Comput. Electron. Agr. 170: 105291, 2020.

Baker N.R.: Chlorophyll fluorescence: a probe of photosynthesis in vivo. - Annu. Rev. Plant Biol. 59: 89-113, 2008.

Balaji G.N., Nandhini V., Mithra S. et al.: Iot based smart crop monitoring in farm land. - Imp. J. Interdiscip. Res. 4: 88-92, 2018.

Barber J., Andersson B.: Too much of a good thing: light can be bad for photosynthesis. - Trends Biochem. Sci. 17: 61-66, 1992.

Bermudez I., Traverso S., Mellia M., Munafò M.: Exploring the cloud from passive measurements: The Amazon AWS case. In: 2013 Proceedings IEEE INFOCOM. Pp. 230-234. IEEE, Turin 2013.

Bulthuis D.A.: Effects of temperature on photosynthesis and growth of seagrasses. - Aquat. Bot. 27: 27-40, 1987.

Campani G., Ribeiro M.P.A., Zangirolami T.C., Lima F.V.: A hierarchical state estimation and control framework for monitoring and dissolved oxygen regulation in bioprocesses. Bioprocess Biosyst. Eng. 42: 1467-1481, 2019.

Chang T.G., Zhu X.G.: Source-sink interaction: a century old concept under the light of modern molecular systems biology. - J. Exp. Bot. 68: 4417-4431, 2017.

Christensen A.J., Srinivasan V., Hart J.C. et al.: Use of computational modeling combined with advanced visualization to develop strategies for the design of crop ideotypes to address food security. - Nutr. Rev. 76: 332-347, 2018.

Dietz K.-J., Schreiber U., Heber U.: The relationship between the redox state of $\mathrm{Q}_{\mathrm{A}}$ and photosynthesis in leaves at various carbon-dioxide, oxygen and light regimes. - Planta 166: 219226, 1985.

Dinar A., Tieu A., Huynh H.: Water scarcity impacts on global food production. - Glob. Food Secur. 23: 212-226, 2019.

Feng S., Fu L., Xia Q. et al.: Modelling and simulation of photosystem II chlorophyll fluorescence transition from darkadapted state to light-adapted state. - IET Syst. Biol. 12: 289293, 2018.

Fernandez-Jaramillo A.A., Duarte-Galvan C., Contreras-Medina L.M. et al:: Instrumentation in developing chlorophyll fluorescence biosensing: A review. - Sensors-Basel 12: 11853-11869, 2012

Fu L., Govindjee G., Tan J., Guo Y.: Development of a minimized model structure and a feedback control framework for regulating photosynthetic activities. - Photosynth. Res. 146: 
213-225, 2020.

Genty B., Briantais J.-M., Baker N.R.: The relationship between the quantum yield of photosynthetic electron transport and quenching of chlorophyll fluorescence. - BBA-Gen. Subjects 990: 87-92, 1989.

Govindjee G., Amesz J., Fork D.C.: Light Emission by Plants and Bacteria. Pp. 638. Academic Press, Orlando 1986.

Guarini J.-M., Moritz C.: Modelling the dynamics of the electron transport rate measured by PAM fluorimetry during rapid light curve experiments. - Photosynthetica 47: 206-214, 2009.

Guo Y., Tan J.: Modeling and simulation of the initial phases of chlorophyll fluorescence from Photosystem II. - BioSystems 103: 152-157, 2011.

Hemming S., de Zwart F., Elings A. et al.: Remote control of greenhouse vegetable production with artificial intelligence greenhouse climate, irrigation, and crop production. Sensors-Basel 19: 1807, 2019.

Iersel M.W., Mattos E., Weaver G. et al.: Using chlorophyll fluorescence to control lighting in controlled environment agriculture. - Acta Hortic. 1134: 427-434, 2016a.

Iersel M.W., Weaver G., Martin M.T. et al.: A chlorophyll fluorescence-based biofeedback system to control photosynthetic lighting in controlled environment agriculture. - J. Am. Soc. Hortic. Sci. 141: 169-176, 2016b.

Juhasova B., Juhas M., Halenar I.: TCP/IP protocol utilisation in process of dynamic control of robotic cell according industry 4.0 concept. - In: 2017 IEEE $15^{\text {th }}$ International Symposium on Applied Machine Intelligence and Informatics. Pp. $000217-$ 000222. SAMI, 2017.

Kannan K., Wang Y., Lang M. et al.: Combining gene network, metabolic and leaf-level models show means to future-proof soybean photosynthesis under rising $\mathrm{CO}_{2}$. - in silico Plants $\mathbf{1}$ : diz008, 2019.

Kennedy D., Norman C.: What don't we know? - Science 309: 75, 2005.

Kim J.Y.: Roadmap to high throughput phenotyping for plant breeding. - J. Biosyst. Eng. 45: 43-55, 2020.

Kramer P.J.: Carbon dioxide concentration, photosynthesis, and dry matter production. - BioScience 31: 29-33, 1981.

Light R.A.: Mosquitto: server and client implementation of the MQTT protocol. - J. Open Source Softw. 2: 265, 2017.

Long S.P., Marshall-Colon A., Zhu X.G.: Meeting the global food demand of the future by engineering crop photosynthesis and yield potential. - Cell 161: 56-66, 2015.

Mahmoud R., Yousuf T., Aloul F., Zualkernan I.: Internet of things (IoT) security: Current status, challenges and prospective measures. - In: $201510^{\text {th }}$ International Conference for Internet Technology and Secured Transactions (ICITST). Pp. 336-341. IEEE, London 2015.

Malik A.W., Rahman A.U., Qayyum T., Ravana S.D.: Leveraging fog computing for sustainable smart farming using distributed simulation. - IEEE Internet Things J. 7: 3300-3309, 2020.

Marshall-Colon A., Long S.P., Allen D.K. et al.: Crops in silico: generating virtual crops using an integrative and multi-scale modeling platform. - Front. Plant Sci. 8: 786, 2017.

Padhi B., Chauhan G., Kandoi D. et al.: A comparison of chlorophyll fluorescence transient measurements, using Handy PEA and FluorPen fluorometers. - Photosynthetica 59: 399-408, 2021.

Pasha S.: ThingSpeak based sensing and monitoring system for IoT with Matlab analysis. - Int. J. New Technol. Res. 2: 1923, 2016.

Pommier C., Cornut G., Letellier T. et al.: Data standards for plant phenotyping: MIAPPE and its implementations. In: 26. Plant and Animal Genome Conference (PAG XXVI), Jan 2018, San Diego, California, United States. Pp. 24 slides. San Diego 2018.

Schansker G., Tóth S.Z., Holzwarth A.R., Garab G.: Chlorophyll $a$ fluorescence: beyond the limits of the $\mathrm{Q}_{\mathrm{A}}$ model. Photosynth. Res. 120: 43-58, 2014.

Shinkarev V.P., Govindjee G.: Insight into the relationship of chlorophyll $a$ fluorescence yield to the concentration of its natural quenchers in oxygenic photosynthesis. - P. Natl. Acad. Sci. USA 90: 7466-7469, 1993.

Sipka G., Magyar M., Mezzetti A. et al.: Light-adapted chargeseparated state of photosystem II: structural and functional dynamics of the closed reaction center. - Plant Cell 33: 12861302, 2021.

Stirbet A., Riznichenko G.Yu., Rubin A., Govindjee: Modeling chlorophyll $a$ fluorescence transient: relation to photosynthesis. - Biochemistry-Moscow 79: 291-323, 2014.

Verdouw C., Sundmaeker H., Tekinerdogan B. et al.: Architecture framework of IoT-based food and farm systems: A multiple case study. - Comput. Electron. Agr. 165: 104939, 2019.

Walker B.J., Busch F.A., Driever S.M. et al.: Survey of tools for measuring in vivo photosynthesis. - In: Covshoff S. (ed.): Photosynthesis. Vol. 1770. Pp. 3-24. Humana Press, New York 2018.

Yu J., Liberton M., Cliften P.F. et al.: Synechococcus elongatus UTEX 2973, a fast growing cyanobacterial chassis for biosynthesis using light and $\mathrm{CO}_{2}$. - Sci. Rep.-UK 5: 8132, 2015.

Zhou W., Li L., Luo M., Chou W.: REST API design patterns for SDN northbound API. - $201428^{\text {th }}$ International Conference on Advanced Information Networking and Applications Workshops. Pp. 358-365. IEEE, Victoria 2014.

Zhu X.G., Govindjee G., Baker N.R. et al:: Chlorophyll a fluorescence induction kinetics in leaves predicted from a model describing each discrete step of excitation energy and electron transfer associated with photosystem II. - Planta 223: 114-133, 2005.

(C) The authors. This is an open access article distributed under the terms of the Creative Commons BY-NC-ND Licence. 